\title{
Sputum microbiome temporal variability and dysbiosis in chronic obstructive pulmonary disease exacerbations: an analysis of the COPDMAP study
}

\author{
Zhang Wang, ${ }^{1}$ Richa Singh, ${ }^{2}$ Bruce E Miller, ${ }^{3}$ Ruth Tal-Singer, ${ }^{3}$ \\ Stephanie Van Horn, ${ }^{4}$ Lynn Tomsho, ${ }^{4}$ Alexander Mackay, ${ }^{2}$ James P Allinson, ${ }^{2}$ \\ Adam J Webb, ${ }^{5}$ Anthony J Brookes, ${ }^{5}$ Leena M George, ${ }^{6}$ Bethan Barker, ${ }^{6}$ \\ Umme Kolsum, ${ }^{7}$ Louise E Donnelly, ${ }^{2}$ Kylie Belchamber, ${ }^{2}$ Peter J Barnes, ${ }^{2}$ \\ Dave Singh, ${ }^{7}$ Christopher E Brightling, ${ }^{6}$ Gavin C Donaldson, ${ }^{2}$ Jadwiga A Wedzicha, ${ }^{2}$ \\ James $R$ Brown, ${ }^{1}$ on behalf of COPDMAP
}

\begin{abstract}
- Additional material is published online only. To view please visit the journal online (http://dx.doi.org/10.1136/ thoraxjnl-2017-210741).

For numbered affiliations see end of article.

Correspondence to Dr James R Brown,

Computational Biology, Target Sciences, Research and Development (R\&D), GlaxoSmithKline (GSK), Collegeville, PA 19426, USA james.r.brown@gsk.com
\end{abstract}

ZW and RS contributed equally.

Received 7 July 2017

Revised 2 November 2017 Accepted 27 November 2017 Published Online First 21 December 2017

Check for updates

To cite: Wang Z, Singh R, Miller BE, et al. Thorax 2018:73:331-338.

\section{ABSTRACT}

Background Recent studies suggest that lung microbiome dysbiosis, the disease associated disruption of the lung microbial community, might play a key role in chronic obstructive pulmonary disease (COPD) exacerbations. However, characterising temporal variability of the microbiome from large longitudinal COPD cohorts is needed to better understand this phenomenon.

Methods We performed a 165 ribosomal RNA survey of microbiome on 716 sputum samples collected longitudinally at baseline and exacerbations from 281 subjects with COPD at three UK clinical centres as part of the COPDMAP consortium.

Results The microbiome composition was similar among centres and between stable and exacerbations except for a small significant decrease of Veillonella at exacerbations. The abundance of Moraxella was negatively associated with bacterial alpha diversity. Microbiomes were distinct between exacerbations associated with bacteria versus eosinophilic airway inflammation. Dysbiosis at exacerbations, measured as significant within subject deviation of microbial composition relative to baseline, was present in $41 \%$ of exacerbations. Dysbiosis was associated with increased exacerbation severity indicated by a greater fall in forced expiratory volume in one second, forced vital capacity and a greater increase in CAT score, particularly in exacerbations with concurrent eosinophilic inflammation. There was a significant difference of temporal variability of microbial alpha and beta diversity among centres. The variation of beta diversity significantly decreased in those subjects with frequent historical exacerbations.

Conclusions Microbial dysbiosis is a feature of some exacerbations and its presence, especially in concert with eosinophilic inflammation, is associated with more severe exacerbations indicated by a greater fall in lung function. Trial registration number Results, NCT01620645.

\section{INTRODUCTION}

Chronic obstructive pulmonary disease (COPD) is characterised by persistent symptoms and impaired lung function as a consequence of small airway obliteration and alveolar destruction and is associated with chronic lung inflammation. ${ }^{1-3}$

\section{Key messages}

What is the key question?

- How does the lung microbial community vary over time within chronic obstructive pulmonary disease (COPD) subjects and how is microbial dysbiosis in exacerbations implicated in disease characteristics?

What is the bottom line?

- Dysbiosis of the sputum microbiome in COPD exacerbations, particularly in concert with eosinophilic inflammation, is associated with a greater decline in lung capacity during the exacerbation event.

Why read on?

- The presented study entails the largest COPD sputum microbiome cohort to date with multiple study centres, aiming at in-depth examination of microbial temporal variability, dysbiosis and disease phenotypes.

Acute exacerbations of COPD are a sudden onset of sustained worsening of these symptoms. Bacteria potentially play a key role in COPD pathogenesis, ${ }^{4} 5$ with respiratory bacterial pathogens such as Haemophilus influenzae, Moraxella catarrhalis and Streptococcus pneumoniae capable of driving host inflammatory responses. ${ }^{6-9}$ Since bacteria frequently interact with each other and respond to altered environmental conditions, the consortium of the lung microbial community, known as the lung microbiome, could be important in the crosstalk between respiratory tract pathogens and host response. ${ }^{1011}$

Emerging studies collectively suggest that the lung microbiome differs between stable and exacerbations in $\mathrm{COPD}^{11-15}$ (for review see Huang et $a l^{16}$ ). For example, Molyneaux et $a l^{12}$ found an increased representation of pathogenic Proteobacteria in particular Haemophilus in exacerbations following rhinovirus infection. Huang et al observed an increase of Proteobacteria during 
exacerbations with a predicted loss of function in maintenance of microbial homeostasis. ${ }^{13}$ Recently, several of us published a longitudinal analysis of the sputum microbiome from 87 subjects from BEAT-COPD cohort. ${ }^{11}$ Our analysis revealed an increased Proteobacteria versus Firmicutes during exacerbations. In addition, we found distinct microbiome composition between bacterial and eosinophilic exacerbations. In light of the heterogeneous nature of COPD exacerbations, the lung microbiome has potential as a biomarker to assist in the precision medicine treatments for specific patient subpopulations with COPD.

Although insightful, results from these previous studies have limitations in terms of understanding microbial dysbiosis during exacerbations, as most of these studies comparing the microbiome at stable and exacerbations involved only one single sampling point of each state. The lung microbiome is temporally dynamic and can vary even in stable state. ${ }^{10}$ Thus the microbial changes during exacerbations are a mixture of both the disease associated disruption of microbial community or dysbiosis and the regular temporal perturbations of the lung microbial composition. Therefore, examining the baseline variation of the lung microbiome is an important first step to more precisely assess the extent of microbial dysbiosis during exacerbations. On the other hand, understanding temporal variability of the lung microbiome within individuals is also important in disease understanding. Disorder of the temporal balance of microbial ecosystem in the respiratory tract could trigger a dysregulated host immune response that results in negative effects on host biology. ${ }^{10}$ Linking microbial temporal variation to disease characteristics and host inflammatory profiles could potentially lead to monitoring and manipulating the stability of airway microbial composition as a therapeutic strategy for COPD.

A finer-grained longitudinal sampling of microbiome at multiple stable and exacerbation visits is necessary to quantitatively measure temporal variability of the microbiome and assess the significance of microbial dysbiosis during exacerbations. Here we describe a longitudinal $16 \mathrm{~S}$ ribosomal RNA (rRNA) gene-based microbiome survey on 716 sputum samples collected sequentially at baseline and exacerbations over a period of up to 2 years duration from 281 subjects with COPD at three UK centres as part of the COPDMAP consortium. This entails one of the largest COPD sputum microbiome cohorts to date aiming at in-depth examination of temporal variability of the microbiome. We provide new insights into temporal changes of the microbiome and its potential implication in disease progression.

\section{MATERIAL AND METHODS Subjects and samples}

Full information on subject inclusion/exclusion criteria, sputum sample collection, microbiome and statistical analyses are provided in the online supplementary appendix. Briefly, sputum samples were collected at multiple longitudinal baseline and exacerbation visits from COPD subjects at three clinical centres: Imperial College London, University of Leicester and University Hospital of South Manchester (hereafter referred to as London, Leicester and Manchester, respectively) as part of the COPDMAP consortium (www.copdmap. org). All sputum samples were immediately stored at $-80^{\circ} \mathrm{C}$ and shipped frozen in batches for analysis. Exacerbations were treated with corticosteroids and antibiotics according to guidelines. ${ }^{17}$ The protocol summary is available at https://clinicaltrials.gov/ (Identifier: NCT01620645).

\section{Microbiome analysis}

For quality control purposes, all DNA extractions, sequencing and data analyses were performed in a single, centralised lab at the GSK R\&D facility in Collegeville, Pennsylvania, USA. Bacterial genomic DNA was extracted from frozen sputum samples using the Qiagen DNA Mini kit (Qiagen, California, USA) as per manufacture protocol. The V4 hypervariable region of the 16S rRNA gene was PCR amplified and sequenced using multiplexed Illumina Miseq platform with the proper controls against reagent contamination as described previously. ${ }^{11}$ Sequencing reads were processed using QIIME pipeline V.1.9. ${ }^{18}$ The default set of criteria was used to remove low quality and chimeric reads. The remaining reads were subject to a close reference operational taxonomic unit (OTU) picking (97\% identity cut-off). Sequence data are deposited at the National Centre for Biotechnology Information Sequence Read Archive (SRP102480).

\section{Statistical analysis}

Exacerbation phenotypes were defined using microbiological and clinical criteria as established previously. ${ }^{12}$ Phenotypes of 146 exacerbations samples were undetermined due to missing data. Partial least squares discriminant analysis (PLS-DA) was performed on exacerbation phenotypes and microbiome and/or clinical data using SIMCA-P (Umetrics, Stockholm, Sweden). ${ }^{19}$ Dysbiosis at exacerbations was measured as the deviation (Z-score) of the first principal coordinate (PC1) of the weighted UniFrac distance for exacerbation samples relative to all baseline PC1s from the same subject. Temporal variability of microbial alpha and beta diversity was measured using the metrics described by Flores et al. ${ }^{20}$ A general linear model (GLM) was constructed between demographic and baseline clinical variables and temporal variability of alpha and beta diversity among subjects. The model was optimised in a stepwise algorithm using the 'step' function in the $\mathrm{R}$ stats package. ${ }^{21}$ The false discovery rate (FDR) method was used to adjust $\mathrm{P}$ values for multiple testing wherever applicable. ${ }^{22}$

\section{RESULTS}

\section{Overview of the COPDMAP sputum microbiome}

Microbial composition was determined for 716 sputum samples collected at baseline and exacerbations from 281 COPDMAP subjects at three centres. The number of samples varies from one to nine per subject (online supplementary figure S1). Demographic and baseline clinical data were recorded for subjects at initiation of sample collection (table 1; online supplementary table S1). A set of 16 clinical and biochemical characteristics were further collected longitudinally (table 2; online supplementary table S2). From DNA sequences of the V4 hypervariable region of the $16 \mathrm{~S}$ rRNA gene, a total of 3784 OTUs were identified using 97\% identity cut-off after rarefaction.

Similar to other sputum or lung microbiome studies, ${ }^{11-15}$ 23-26 the vast majority of OTUs belonged to Proteobacteria (52.3\%), Firmicutes (28.7\%), Bacteroidetes (15.0\%) and Actinobacteria $(1.9 \%)$ at the phylum level (online supplementary table S3 and figure S2). At the genus level, Haemophilus (25.8\%) was most abundant across all samples, followed by Veillonella (15.8\%) and Prevotella (13.2\%). Other common genera in the airway such as Streptococcus (4.4\%) and Moraxella (4.0\%) were also among the most abundant genera identified. As a quality control for sample processing and sequence analyses, an additional aliquot of sputum was collected as duplicates for 11 samples from the same subject at the same visit. Duplicates all 


\begin{tabular}{|c|c|}
\hline $\begin{array}{l}\text { Demographic and } \\
\text { baseline features }\end{array}$ & All subjects $(n=281)^{*}$ \\
\hline Gendert & $\begin{array}{l}\text { Male: } 187(70.3 \%) \text {, } \\
\text { Female: } 79(29.7 \%)\end{array}$ \\
\hline Age $\ddagger$ & $70(8.1)$ \\
\hline BMI & $27.2(5.4)$ \\
\hline Baseline GOLD status & $\begin{array}{l}\text { 1: } 30(11.4 \%), 2: 132(50.2 \%), 3: 78 \\
(29.7 \%), 4: 23(8.7 \%)\end{array}$ \\
\hline Treatment§ & $\begin{array}{l}\text { Antibiotics: } 38 \text { (15.3\%), Steroids: } 9 \\
\text { (3.6\%), Both: } 202(81.1 \%)\end{array}$ \\
\hline Number of cigarette packs per yearq & $47(30)$ \\
\hline Number of exacerbation per yearq & $1.1(1.6)$ \\
\hline Baseline $\mathrm{FEV}_{1}$ & $1.5(0.6)$ \\
\hline Baseline $\mathrm{FEV}_{1} \%$ & $56.3(18.9)$ \\
\hline Baseline $\mathrm{FEV}_{1}$ predicted & $2.6(0.5)$ \\
\hline Baseline FVC & $2.9(1.0)$ \\
\hline Baseline $\mathrm{FEV}_{1} / \mathrm{FVC}$ ratio & $0.5(0.1)$ \\
\hline CAT score & $18.7(7.3)$ \\
\hline CES-D score 9 & $10(13)$ \\
\hline SGRQ total score & $47.4(18.2)$ \\
\hline \multicolumn{2}{|c|}{$\begin{array}{l}\text { *15 subjects were missing any demographic or clinical data. } \\
\text { †Categorical data present as number (proportion). } \\
\text { †Continuous data present as mean (SD) unless stated below. } \\
\text { §The numbers represent exacerbation events and thus include subjects with more } \\
\text { than one exacerbation. } \\
\text { IMedian (IQR). } \\
\text { BMI, body mass index; CAT, COPD Assessment Test; CES-D, Center for } \\
\text { Epidemiologic Studies Depression; FEV }{ }_{1} \text {, forced expiratory volume in one second; } \\
\text { FVC, forced vital capacity; GOLD, Global Initiative for Chronic Obstructive Lung } \\
\text { Disease; SGRQ, St. George's Respiratory Questionnaire. }\end{array}$} \\
\hline
\end{tabular}

had low UniFrac distance and were highly similar in microbial composition (online supplementary figure S3).

Overall, the microbiome composition was similar between baseline and exacerbation samples with a small significant decrease of Veillonella at exacerbations (repeated measures analysis of variance (ANOVA), FDR-adjusted (adj.) $\mathrm{P}=0.042$ ) (figure 1A). The microbiome composition was similar among centres with a significantly higher alpha diversity in the London cohort (online supplementary figure S4A). Within each centre, there was a significant decrease of alpha diversity (Shannon, repeated measures ANOVA, $\mathrm{P}=1.1 \mathrm{e}-4)$ and a non-significant increase of Moraxella (repeated measures ANOVA, adj. $\mathrm{P}=0.092$ ) at Leicester (online supplementary figure S4B). A strong negative correlation was found between the abundance of Moraxella and alpha diversity for all samples (Shannon, $\mathrm{R}=-0.445$, adj. $\mathrm{P}<9.6 \mathrm{e}-14$, figure $1 \mathrm{~B})$.

Similar to previously observed ${ }^{11}$ distinct microbial populations were found in bacterial and eosinophilic exacerbations, with a significantly decreased alpha diversity (Shannon, t-test $\mathrm{P}=0.008$ ) and significantly increased proportion of Proteobacteria (t-test, adj. $\mathrm{P}=0.001$ ) versus Bacteroidetes ( $\mathrm{t}$-test, adj. $\mathrm{P}=0.002$ ) in bacterial exacerbations compared with eosinophilic exacerbations (figure 1C,D and online supplementary figure S5A). An improvement in predicting the two phenotypes was observed according to PLS-DA by combining the clinical and microbiome datasets versus using the clinical data only (online supplementary figure S5B). Within individual centres, this trend was more pronounced for Leicester samples than those of London or Manchester (online supplementary figure S5C).

We performed multivariate analysis to identify clinical factors significantly associated with microbial alpha and beta diversity. Among all clinical variables, $\mathrm{C}$ reactive protein (CRP), a known inflammatory marker for COPD prognosis, ${ }^{27}$ was the most significant factor correlated with both alpha diversity

Table 2 Major longitudinal clinical features at baseline and exacerbations of all samples

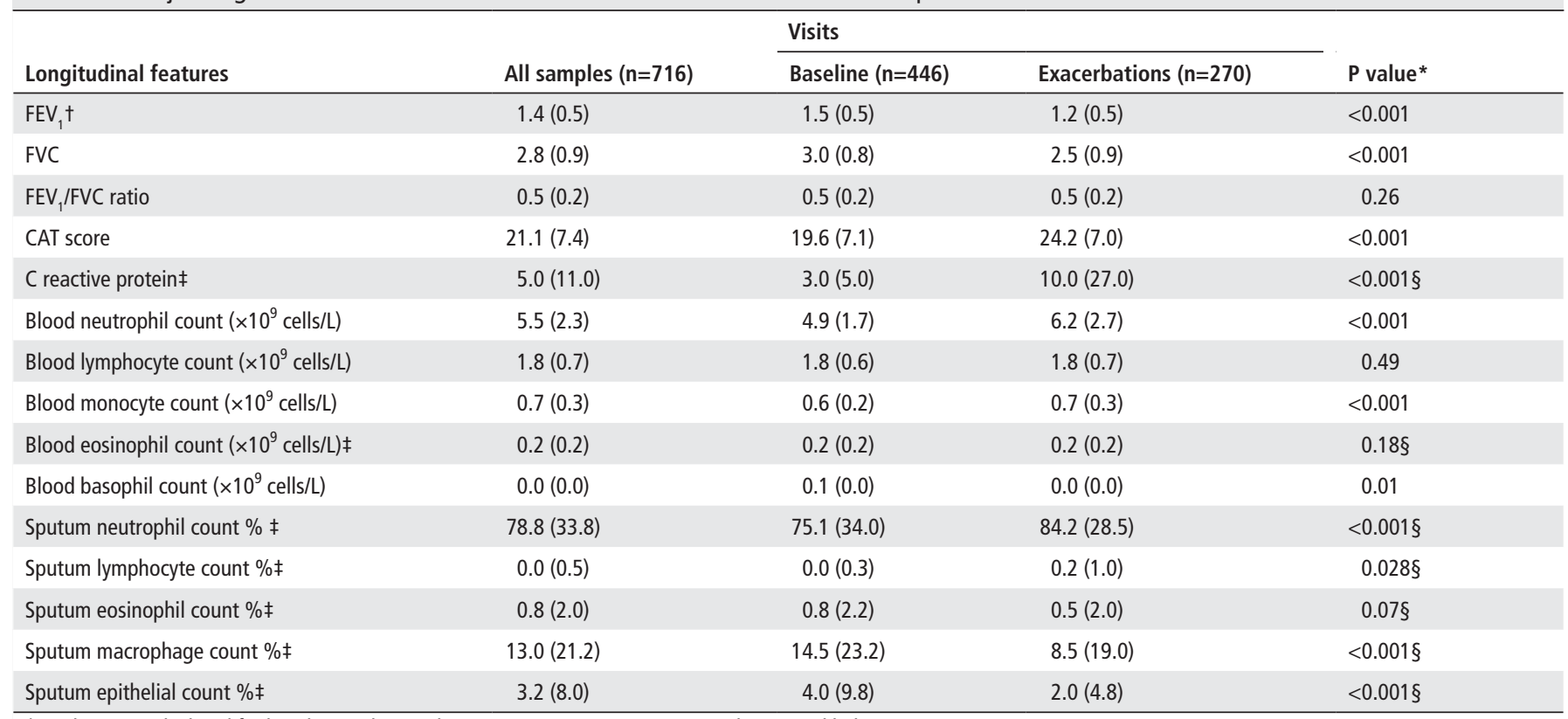

*P value was calculated for baseline and exacerbations comparison using t-test unless stated below.

tData present as mean (SD) unless stated below.

¥Median (IQR).

$\S M a n n-$ Whitney-Wilcoxon Test.

$\mathrm{FEV}_{1}$, forced expiratory volume in one second; FVC, forced vital capacity. 
A

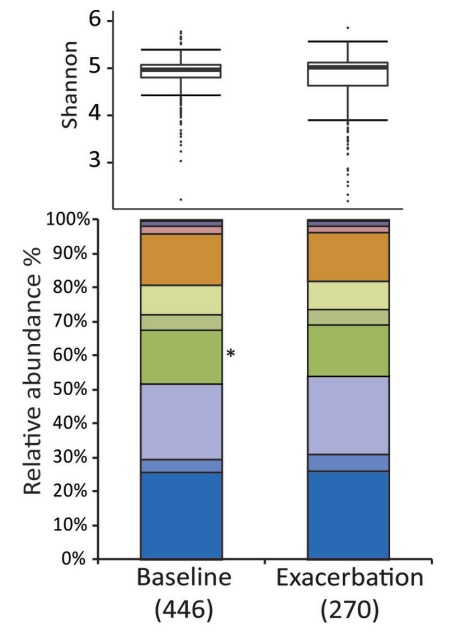

$\square$ Others

口Fusobacteria

$\square$ Actinobacteria

$\square$ Bacteroidetes

$\square$ Firmicutes Others

$\square$ Firmicutes Streptococcus

$\square$ Firmicutes Veillonella

$\square$ Proteobacteria Others

$\square$ Proteobacteria Moraxella

$\square$ Proteobacteria Haemophilus
B
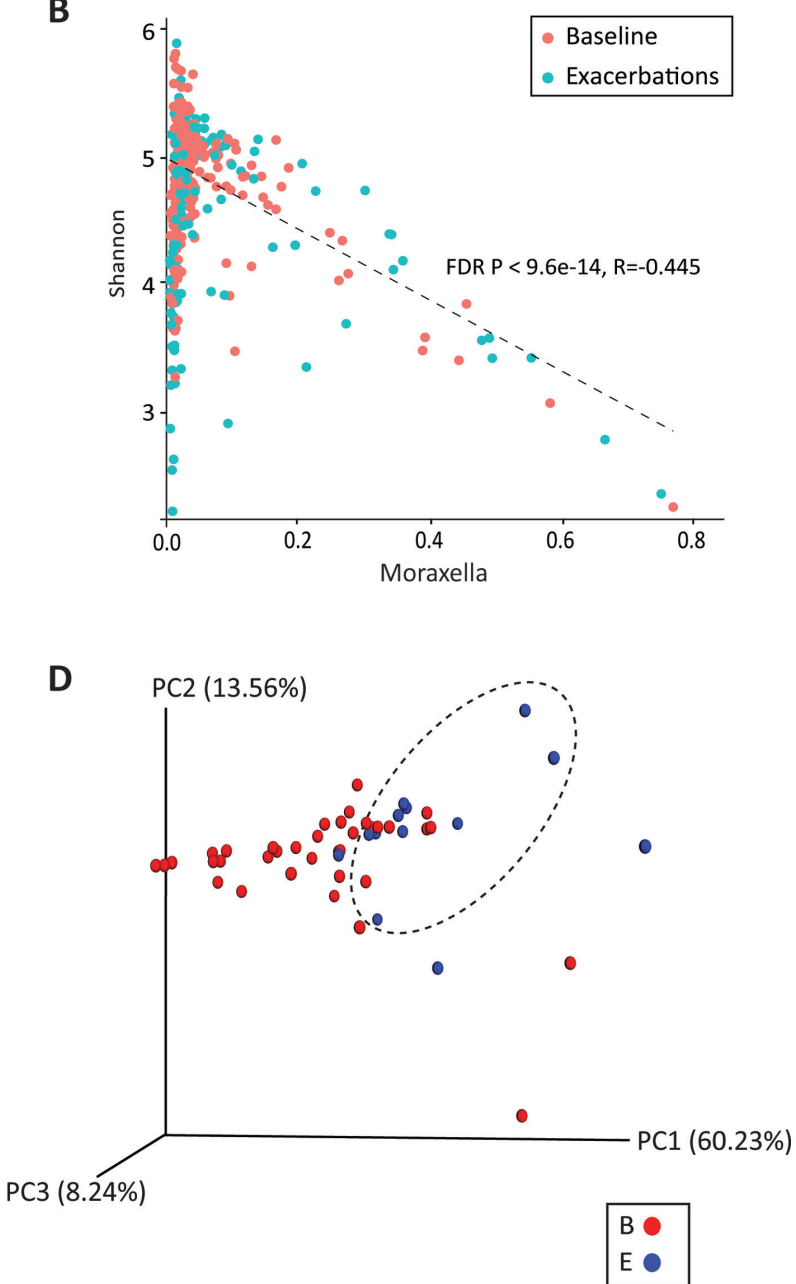

Figure 1 Baseline and exacerbation microbiome profiles across centres. (A) Alpha diversity (Shannon) and compositions of major phyla and genera in samples at baseline and exacerbations. (B) Correlation between alpha diversity (Shannon) and relative abundance of Moraxella. Each dot represents a sample coloured by baseline or exacerbations. (C) Alpha diversity (Shannon) and composition of major phyla and genera in exacerbation samples of different exacerbation phenotypes. (D) PCoA showing distinct clustering of samples with bacterial and eosinophilic exacerbations.

The number of samples is indicated in the parenthesis under each subgroup in the bar chart. Error bars are within $1.5 \mathrm{IQR}$ of the upper and lower quartiles. ${ }^{* * *}$ adj. $\mathrm{P}<0.001$; ${ }^{* *}$ adj. $\mathrm{P}<0.01$; ${ }^{*}$ adj. $\mathrm{P}<0.05$. B, bacterial; $\mathrm{BE}$, bacterial and eosinophilic; BV: bacterial and viral; E, eosinophilic; FDR, false discovery rate; Pauci, pauci-inflammatory; PC1, first principal coordinate; PCoA, principal coordinate analysis; $\mathrm{V}$, viral.

(Shannon, $\mathrm{P}<0.01$; online supplementary figure $\mathrm{S} 6$ ) and beta diversity at the phylum level among all samples (online supplementary table S4). No factors significantly predicted variation at the genus and OTU levels. CRP was also significantly associated with alpha and beta diversity of the predicted functional profiles of the sputum microbiome using the software PICRUSt $^{28}$ (online supplementary table S5).

\section{Increased disease severity in exacerbations with dysbiosis}

To explore variation of the sputum microbiome over time, we plotted the PC1 of the weighted UniFrac distance for all samples within each subject as a proxy for their microbial compositions, as it explains $49.0 \%$ of the total beta diversity (figure 2A). Only subjects with at least two baseline and one exacerbation samples were included. Visual inspection of the plot revealed a deviation of PC1 for many exacerbation samples relative to baseline samples from the same subject (figure 2A; online supplementary figure S7A), indicating specific exacerbations were particularly susceptible to alternation of microbial composition or dysbiosis. In comparison, the sputum microbiome was much less variable among baseline samples. This is supported by a significantly increased within subject SD of PC1 (paired t-test, $\mathrm{P}=6.7 \mathrm{e}-4$ ) combining baseline and exacerbation samples compared with baseline samples only, with the most profound changes at the Leicester centre (online supplementary figure S7B).

Having assessed temporal variability of the sputum microbiome at baseline, we measured the dysbiosis of exacerbation as a Z-score that measures how much its PC1 deviated from all baseline PC1s from the same individual. A total of 49 exacerbations (out of 119 exacerbations with a Z-score, 41.2\%) were identified as in significant dysbiosis state with an absolute Z-score greater than $2(\mathrm{P}<0.05$, figure $2 \mathrm{~A}$; online supplementary figure $\mathrm{S7C}$ ). In most of these exacerbations, the sputum microbiome shifted from a balanced composition to a more biased one predominated by one or a few taxa with a decreased alpha diversity (online supplementary figure S8). Bacterial genera of Veillonella, Cronobacter and Haemophilus were among the key taxa associated with the dysbiosis (online supplementary figure S9). Across all exacerbation subtypes, bacterial 

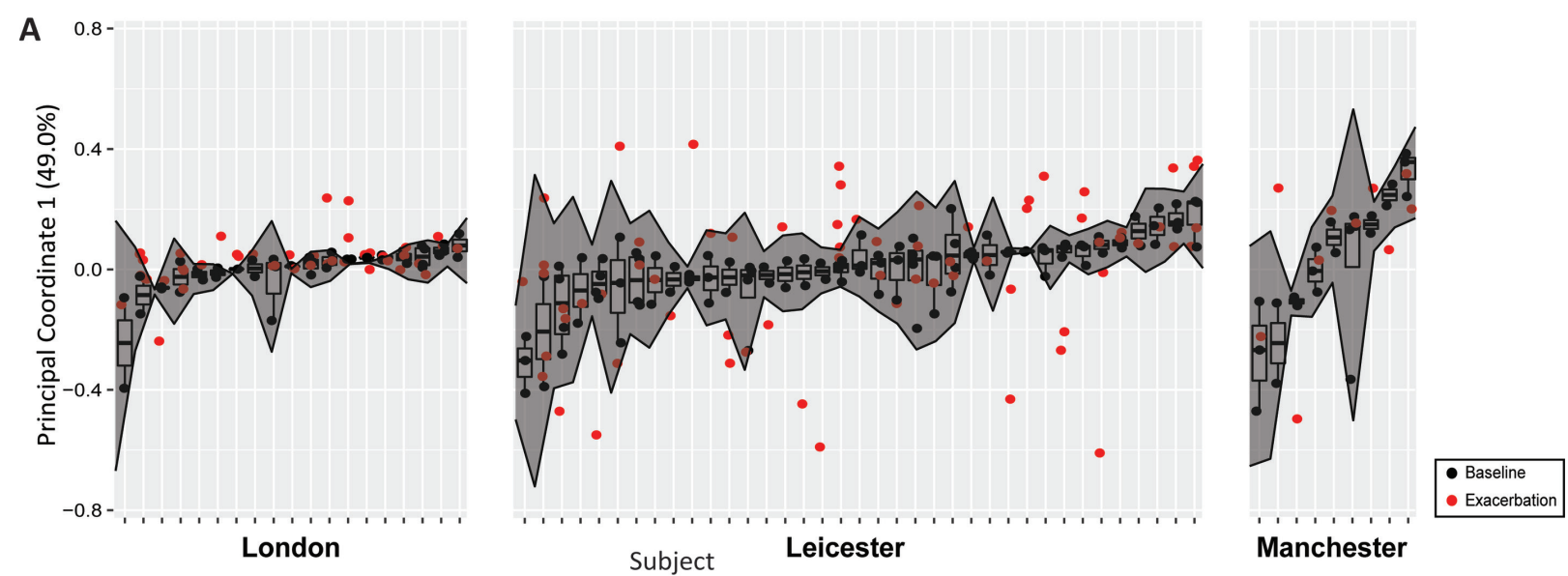

B
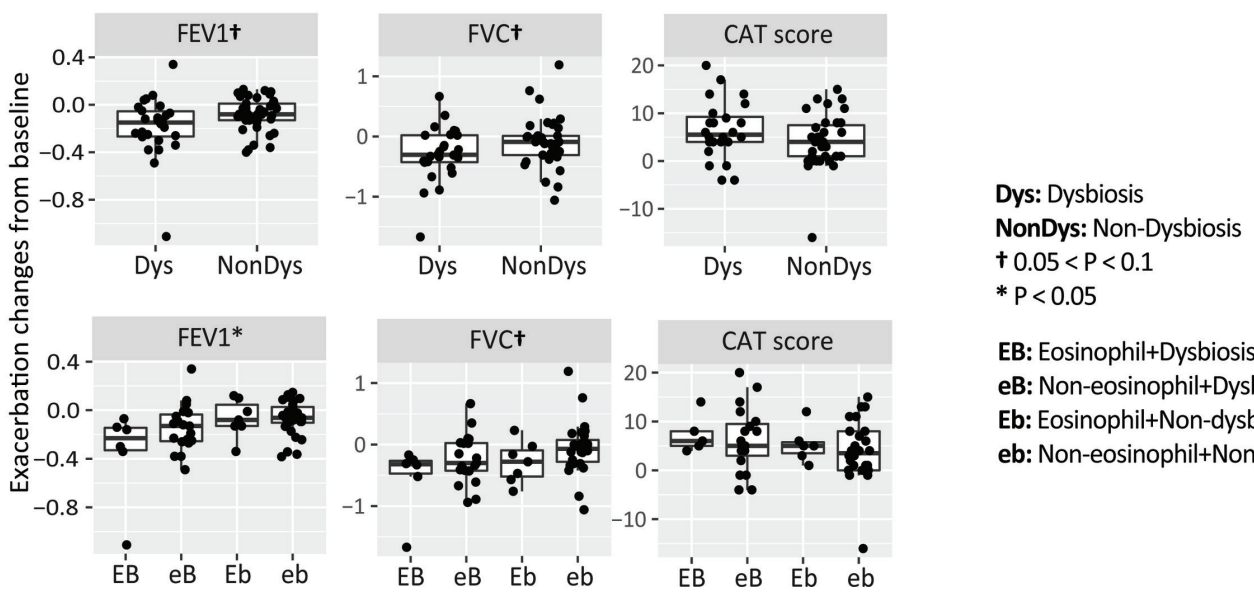

EB: Eosinophil+Dysbiosis eB: Non-eosinophil+Dysbiosis Eb: Eosinophil+Non-dysbiosis eb: Non-eosinophil+Non-dysbiosis

Figure 2 Dysbiosis of the sputum microbiome. (A) Scatter plot of the PC1 of all samples within each subject at each centre. Only subjects with at least two baseline and one exacerbation samples were included. Exacerbation samples are highlighted in red. Box-whisker plots indicate the distribution of baseline PC1s within each subject. The confidence bands indicate the $95 \% \mathrm{Cl}$ for the mean baseline PC1s within each subject. Exacerbations outside the confidence bands are the ones with significant dysbiosis (absolute Z-score $>2, \mathrm{P}<0.05$ ). (B) Box-whisker plots showing changes of FEV ${ }_{1}$ FVC and CAT score between dysbiosis and non-dysbiosis exacerbations and among four subgroups of exacerbations classified by dysbiosis and blood eosinophils level. Error bars are within 1.5 IQR of the upper and lower quartiles. FEV ${ }_{1}$, forced expiratory volume in one second; FVC, forced vital capacity; PC1, first principal coordinate.

exacerbations had the highest number of dysbiosis events than other subtypes (online supplementary figure S7C), with the caveat that phenotype could not be defined for 21 of the 49 exacerbations due to missing data.

For exacerbations with or without significant dysbiosis, we compared the exacerbation severity determined by change in lung function and symptoms relative to the last baseline measurement. We found a non-significantly greater decrease in forced expiratory volume in one second $\left(\mathrm{FEV}_{1}\right)$ and forced vital capacity (FVC) and a greater increase in CAT score for exacerbations with dysbiosis compared with those without (figure $2 \mathrm{~B}$ ). Such trends were overall consistent within each centre, except for a reversal trend of FVC in Manchester which has a smaller sample size (online supplementary figure S10A). Also, the exacerbation Z-score was positively correlated, although non-significantly, with changes of $\mathrm{FEV}_{1}$ and FVC, and negatively correlated with change of CAT score (online supplementary figure S10B), suggesting that the more dysbiotic the exacerbation was, the more severe the clinical outcome could possibly be. As eosinophil abundance is another important factor for COPD exacerbations, we reclassified exacerbations according to both the dysbiosis and blood eosinophil indices. Doing so revealed four subgroups of exacerbations where dysbiosis and/or high blood eosinophil level $\left(>3 \times 10^{8}\right.$ cells/L) are the predominant features. Exacerbations with both dysbiosis and high eosinophil level had the greatest changes of $\mathrm{FEV}_{1}$ (statistically significant, ANOVA $\mathrm{P}=0.02$ ), FVC and CAT score, whereas exacerbations with neither dysbiosis nor high eosinophils level were associated with the least of such changes (figure 2B).

\section{Exacerbation frequency associated with temporal variability of the sputum microbiome}

We next sought to quantify temporal variability of the sputum microbiome within subjects using the metrics described by Flores et al. $^{20}$ Only subjects with at least three samples were included. The variability of microbial alpha diversity was denoted as the coefficient of variation of Shannon for samples within each subject. The variability of beta diversity was calculated as the median of pairwise UniFrac distances for samples within each subject. A wide range of temporal variability of alpha and beta diversity was observed across subjects (figure 3A). We noted that there was a significantly lower variation of alpha and beta diversity among London subjects than Leicester or Manchester ones ( $t$-test, $\mathrm{P}<0.001)$. As expected, both variations of alpha and beta diversity were significantly 
higher in subjects with dysbiosis exacerbations than those without (t-test, $\mathrm{P}<0.01)$.

We constructed a GLM to look for clinical characteristics associated with temporal variability of the sputum microbiome. A set of 14 demographic and baseline clinical variables were included for each subject. Centre, $\mathrm{FEV}_{1} / \mathrm{FVC}$ ratio and number of exacerbations per year (prior to the sampling visit herein called historical exacerbations) were significant factors for the variation of alpha diversity across all subjects (table 3 ). When reconstructing GLM for each centre, number of historical exacerbations per year was significant for London and Leicester subjects. In addition, centre and historical number of exacerbations per year were significantly associated with beta diversity variation across all subjects (table 2). A continuous decreasing trend of historical number of exacerbations per year was observed towards subjects with greater variation of beta diversity (figure 3B). Likewise, a continuous decreasing trend of beta diversity variation was observed towards subjects with higher historical number of exacerbations per year (ANOVA, $\mathrm{P}<0.05$, figure $3 \mathrm{C}$ ). Similar trends were observed within each centre (online supplementary figure S11) and for temporal variability of baseline microbiomes only (online supplementary figure S12), although the association of baseline microbiome variability was not statistically significant (variability of beta diversity: $\mathrm{P}=0.088$, variability of alpha diversity: $\mathrm{P}=0.249)$.

\section{DISCUSSION}

Culture-independent analyses have uncovered a previously unappreciated complexity of the lung bacterial community that has reshaped our understanding of COPD aetiology. ${ }^{11-15}$ 23-26 Our study reveals a diverse sputum microbiome among the COPDMAP subjects and further validates the association of microbiome with specific exacerbation phenotypes. We also show in-depth temporal variation of the sputum microbial community within subjects and identified potential new relationships of the microbiome variation with patient disease progression.

One advantage of our study is the longitudinal sampling at multiple baseline and exacerbation visits compared with most previous studies where a single snapshot of exacerbations was taken. It is well appreciated that the lung microbiome is inherently variable shaped by the balance of ecological factors like microbial immigration and elimination. ${ }^{10}$ During exacerbations, the balance goes awry with dysregulated host immune response and inflammation leading to further microbial changes or dysbiosis. Therefore, to explicitly determine the extent of disease

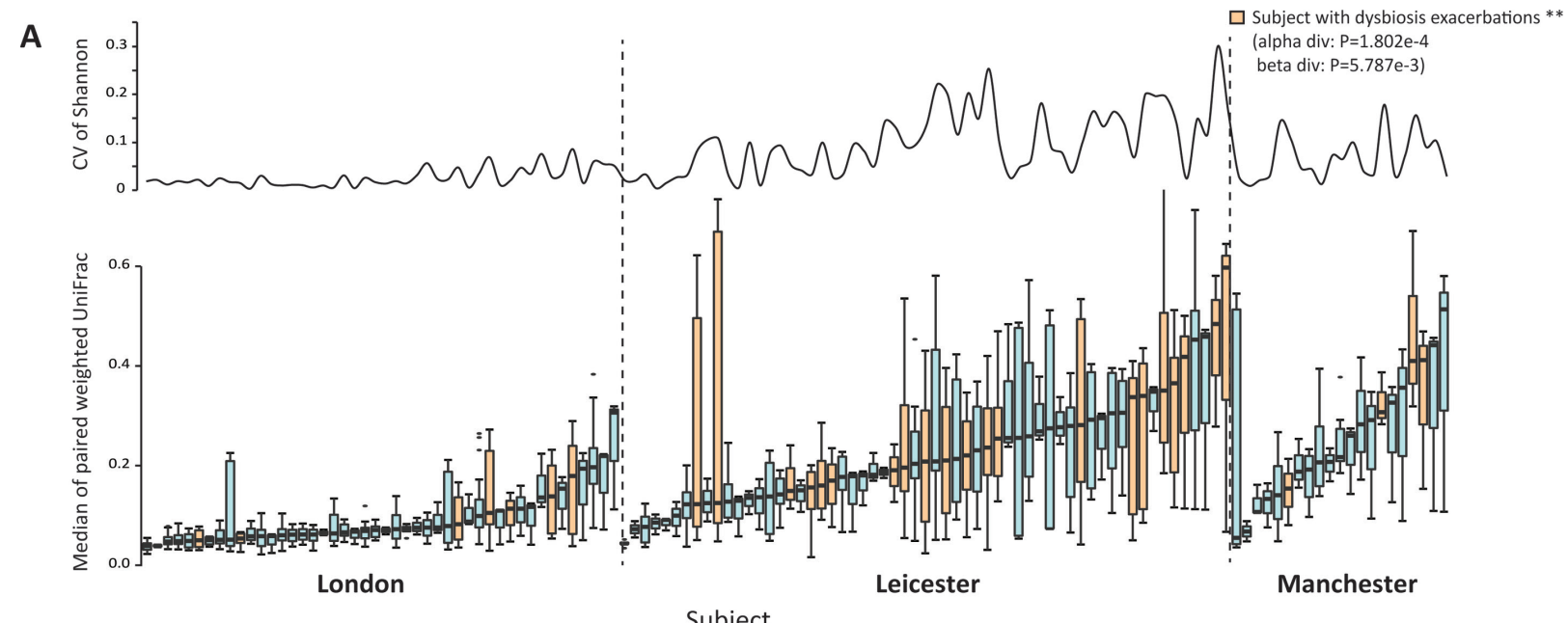

Subject

B

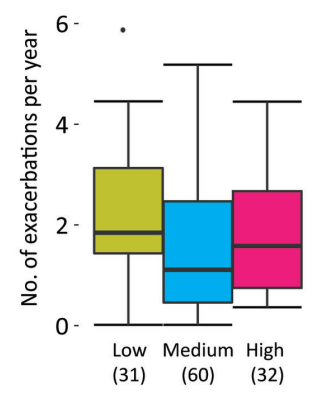

Variation of alpha diversity * Variation of beta diversity *** $(P=0.0427)$

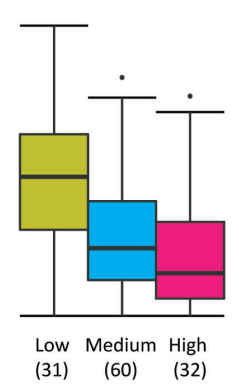

$(\mathrm{P}=1.65 \mathrm{e}-5)$
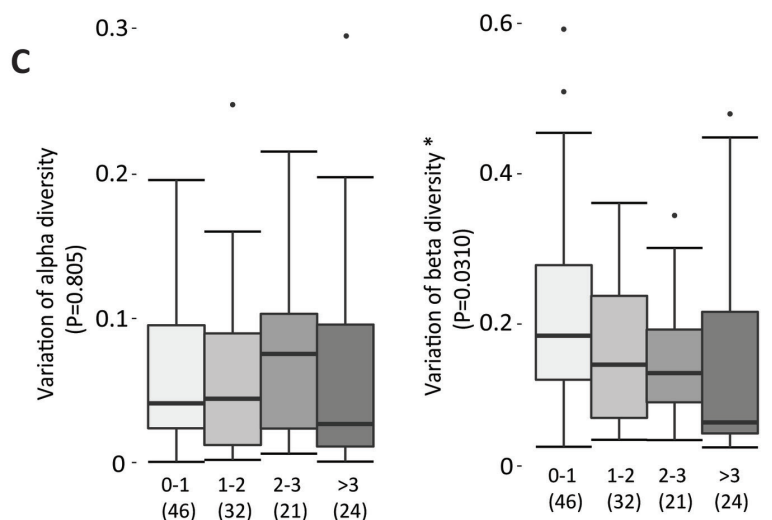

Figure 3 Temporal variability of the sputum microbiome. (A) Temporal variability of microbial alpha (coefficient of variation of Shannon) and beta diversity (median of pairwise weighted UniFrac distances) for each subject. Only subjects with at least three samples were included. Subjects with dysbiosis exacerbations are highlighted in yellow. (B) Box-whisker plots showing exacerbation frequency of subjects within different quartile groups of temporal variability of alpha and beta diversity, with the first quartile defined as 'low', the second and third quartiles as 'medium' and the fourth quartile as 'high'. (C) Box-whisker plots showing temporal variability of alpha and beta diversity in subjects with different classes of exacerbation frequency. The number of samples is indicated in the parenthesis under each subgroup in the box-whisker plot. Error bars are within $1.5 \mathrm{IQR}$ of the upper and lower quartiles. Analysis of variance test for temporal variability of alpha and beta diversity: ${ }^{* * *}$ adj. $P<0.001 ;{ }^{* *}$ adj. $P<0.01 ;{ }^{*}$ adj. $P<0.05$. 
Table 3 List of demographic and baseline clinical variables significantly associated with temporal variability of microbial alpha and beta diversity among subjects

\begin{tabular}{|c|c|c|c|c|c|c|c|c|}
\hline \multirow[b]{2}{*}{ Temporal variability } & \multicolumn{4}{|c|}{ Alpha diversity (Shannon) } & \multicolumn{4}{|c|}{ Beta diversity (weighted UniFrac distance) } \\
\hline & All & London & Leicester & Manchester & All & London & Leicester & Manchester \\
\hline Historical number of exacerbations per year & 0.01 * & $0.01^{*}$ & $3 e-4^{*}$ & 0.32 & $0.01^{*}$ & $0.03^{*}$ & 0.47 & 0.21 \\
\hline CES-D score & 0.50 & 0.03 & 0.35 & 0.68 & 0.44 & 0.32 & 0.63 & $0.01^{*}$ \\
\hline Packs of cigarette per year & 0.40 & 0.67 & 0.31 & 0.38 & $0.07 \dagger$ & 0.50 & $0.02^{*}$ & 0.58 \\
\hline Age & 0.54 & $0.03^{*}$ & 0.38 & 0.23 & 0.70 & 0.37 & 0.99 & 0.26 \\
\hline SGRQ total score & 0.61 & 0.92 & 0.80 & 0.38 & 0.33 & 0.44 & 0.40 & $0.03^{*}$ \\
\hline Centre & $2.6 \mathrm{e}-12^{*}$ & NA & NA & NA & $1.8 \mathrm{e}-10^{*}$ & NA & NA & NA \\
\hline
\end{tabular}

$P$ values are indicated for variables in the model.

* Significant variables.

†Variables not statistically significant but present in the model.

BMI, body mass index; FEV $_{1}$, forced expiratory volume in one second; FVC, forced vital capacity.

associated dysbiosis one would need to first distil the normal perturbations of microbial composition. Our study underscores the importance of considering temporal variability of the microbiome in understanding the significance of microbial dysbiosis in COPD exacerbations.

From assessing temporal variation of the sputum microbiome, we identified a subset of exacerbation events in which significant dysbiosis is a feature. In these exacerbations, the microbiome composition shifted from a highly diverse microbial community to a less diverse one characterised by the predominance of only one or few genera. These dysbiosis exacerbations appear to be the main source of microbial temporal variation and are associated with a greater worsening of health status and decrease of lung capacity. To our knowledge, this is the first evidence to suggest that respiratory dysbiosis is associated with increased exacerbation severity in COPD, although the strength of this association is weak and needs to be further validated in additional cohorts and by other measures of disease severity. Altered environmental conditions in exacerbations could disturb the composition of the lung microbial community, ${ }^{29} 30$ which in turn elicit a dysregulated host immune response through bacterial metabolites and virulent factors, resulting in a sustained damage cycle with an accelerated decline in lung function. ${ }^{31}$ Whether dysbiosis is the cause or consequence of the increased exacerbation severity and how this imbalance is implicated in host inflammatory pathways are new questions that will impact on how we understand and treat COPD exacerbations.

It has been recently emphasised that not all COPD exacerbations are the same. ${ }^{32}$ Our results suggest the existence of subgroups of exacerbations associated with or without significant microbial dysbiosis or increased eosinophilia. Importantly, these subgroups likely reflect fundamental differences in their immunopathogenesis driving the exacerbations and therefore might require alternative therapeutic approaches. Interestingly, the most severe exacerbations were observed in the small subgroup that had evidence of bacterial dysbiosis in concert with eosinophilic inflammation. It is possible that this group might require interventions such as antibiotics and steroids (ie, prednisolone) to target both bacteria and eosinophilic inflammation whereas in contrast those without bacterial dysbiosis or eosinophilic inflammation might not require these therapies. Our results perhaps establish a new paradigm in stratifying COPD exacerbations according to dysbiosis and eosinophil measurements, which could be informative guiding future personalised therapies. Further efforts in identifying biomarkers for these subgroups in larger populations could help refine exacerbation subtypes towards phenotype-specific clinical management.

We found a significantly decreased historical exacerbation frequency in subjects with higher temporal variation of the microbial beta diversity. In COPD, there is a subset of frequent exacerbators that are particularly susceptible to recurrent exacerbations independent of other risk factors. ${ }^{33} 34$ Thus, low temporal variability of the sputum microbiome might come as a predicative factor for the frequent exacerbator phenotypes. We observed that a rise in exacerbation frequency was associated with a decline in the variation of microbiome beta diversity. This trend was statistically significant in the analysis of all microbiome samples but non-significant for that of a subset comprised of only baseline samples. Therefore, the predictability of sputum microbiome variability for exacerbation frequency still warrants further validation in larger longitudinal cohort studies.

An important novelty of our study is that there were three unique study centres. All samples were processed in a central lab, which minimises microbiome variation due to differences in experimental protocols. Interestingly, there was a significant difference in temporal variability of the microbiome among subjects in the three centres, even though their overall microbiome profiles were highly similar. Factors accounting for the among-centre variation could include differences in the frequency of clinical visits and compliance with medications, although we lack the comparative data across centres to suggest specific causes. Our study suggests that the impact of demographics and clinical procedures on the lung microbial community needs to be broadly considered in future studies.

Our study has several caveats. First, only a proportion of the bacterial 16S rRNA gene was sequenced to characterise the microbial population both here and in previous lung microbiome studies. ${ }^{11-14}$ Thus, the resolution is insufficient when it comes to species-level characterisation of the microbiome, whereas ecological and functional interaction of individual species or strains could be important in the underlying disease aetiology. Second, despite a large cohort size, longitudinal sampling remains relatively sparse for many subjects with variation in the timing of their sampling visits. Further efforts on characterising respiratory tract metagenomes in a more regularly and intensively followed patient cohort together with host multiomics profiling 
would promise to bring in a more comprehensive picture of the intrinsic variability of the lung microbiome and its implications in disease heterogeneity.

In summary, our study revealed a temporally dynamic sputum microbiome in subjects with COPD in which microbial dysbiosis in exacerbations, particularly in concert with eosinophilic inflammation, was associated with increased exacerbation severity. Our findings underscore the importance of considering temporal variability of the sputum microbiome in COPD heterogeneity and its potential as a biomarker towards more precise treatment of COPD.

\section{Author affiliations}

${ }^{1}$ Computational Biology, Target Sciences, Research and Development (R\&D), GlaxoSmithKline, Collegeville, Pennsylvania, USA

${ }^{2}$ National Heart and Lung Institute, Imperial College London, London, UK ${ }^{3}$ Respiratory Therapy Area Unit, R\&D, GlaxoSmithKline, King of Prussia, Pennsylvania, USA

${ }^{4}$ Target and Platform Validation, Target Sciences, GlaxoSmithKline, Collegeville, Pennsylvania, USA

${ }^{5}$ Department of Genetics and Genome Biology, University of Leicester, Leicester, UK ${ }^{6}$ Institute for Lung Health, University of Leicester, Leicester, UK

${ }^{7}$ Centre for Respiratory Medicine and Allergy, University of Manchester and University Hospital of South Manchester, Manchester, UK

Acknowledgements We thank the patients for their participation in this study. Contributors ZW, RS, BEM, RT-S, LED, KB, PJB, DS, CEB, GCD, JAW and JRB contributed to the study conception and design. AM, JPA, AJW, AJB, LMG, BB, UK, LED, KB, DS, CEB, GCD and JAW coordinated the collection of sputum samples and clinical data. SVH and LT performed microbiome DNA purification and sequencing. ZW performed microbiome computational analyses. AJB performed other statistical analysis. ZW wrote the initial draft of the manuscript with additional content provided by RS, RT-S, PJB, CEB and JRB and critical revisions from all authors. All authors read and approved the final version of the manuscript.

Funding COPD MAP (MRC/ABPIInflammation and Immunology Initiative) was funded by the Medical Research Council (UK), Additional supportfor this study was provided by GSKand the Leicester NIHR BRC, University of Leicester, Leicester,UK. This article presentsindependent research funded by the National Institute for Health Research(NIHR)

Disclaimer The views expressed are those of the authors and not necessarily those of the NHS, the NIHR or the Department of Health.

Competing interests ZW, BEM, RT-S, SVH, LT and JRB were employees and shareholders in GlaxoSmithKline PLC at the time of this study. Other authors have no competing interest to declare.

Ethics approval Imperial College London, University of Leicester and University of Manchester Research Ethics Committee.

Provenance and peer review Not commissioned; externally peer reviewed.

(c) Article author(s) (or their employer(s) unless otherwise stated in the text of the article) 2018. All rights reserved. No commercial use is permitted unless otherwise expressly granted.

\section{REFERENCES}

1 Lopez AD, Shibuya K, Rao C, et al. Chronic obstructive pulmonary disease: current burden and future projections. Eur Respir J 2006;27:397-412.

2 Franklin W, Lowell FC, Michelson AL, et al. Chronic obstructive pulmonary emphysema; a disease of smokers. Ann Intern Med 1956:45:268-74.

3 Taraseviciene-Stewart L, Douglas IS, Nana-Sinkam PS, et al. Is alveolar destruction and emphysema in chronic obstructive pulmonary disease an immune disease? Proc Am Thorac Soc 2006:3:687-90.

4 Erkan L, Uzun O, Findik S, et al. Role of bacteria in acute exacerbations of chronic obstructive pulmonary disease. Int J Chron Obstruct Pulmon Dis 2008:3:463-7.

5 Sethi S, Murphy TF. Bacterial infection in chronic obstructive pulmonary disease in 2000: a state-of-the-art review. Clin Microbiol Rev 2001:14:336-63.
6 Miravitlles M, Espinosa C, Fernández-Laso E, et al. Relationship between bacterial flora in sputum and functional impairment in patients with acute exacerbations of COPD. Study group of bacterial infection in COPD. Chest 1999;116:40-6.

7 Ball P. Epidemiology and treatment of chronic bronchitis and its exacerbations. Chest 1995;108(2 Suppl):S43-52.

8 Soler $\mathrm{N}$, Torres A, Ewig S, et al. Bronchial microbial patterns in severe exacerbations of chronic obstructive pulmonary disease (COPD) requiring mechanical ventilation. Am J Respir Crit Care Med 1998;157(5 Pt 1):1498-505.

9 Monsó E, Ruiz J, Rosell A, et al. Bacterial infection in chronic obstructive pulmonary disease. A study of stable and exacerbated outpatients using the protected specimen brush. Am J Respir Crit Care Med 1995;152(4 Pt 1):1316-20.

10 Dickson RP, Martinez FJ, Huffnagle GB. The role of the microbiome in exacerbations of chronic lung diseases. Lancet 2014;384:691-702.

11 Wang Z, Bafadhel M, Haldar K, et al. Lung microbiome dynamics in COPD exacerbations. Eur Respir J 2016:47:1082-92.

12 Molyneaux PL, Mallia P, Cox MJ, et al. Outgrowth of the bacterial airway microbiome after rhinovirus exacerbation of chronic obstructive pulmonary disease. Am J Respir Crit Care Med 2013;188:1224-31.

13 Huang YJ, Sethi S, Murphy T, et al. Airway microbiome dynamics in exacerbations of chronic obstructive pulmonary disease. J Clin Microbiol 2014;52:2813-23.

14 Millares L, Ferrari R, Gallego M, et al. Bronchial microbiome of severe COPD patients colonised by Pseudomonas aeruginosa. Eur J Clin Microbiol Infect Dis 2014;33:1101-11.

15 Huang YJ, Kim E, Cox MJ, et al. A persistent and diverse airway microbiota present during chronic obstructive pulmonary disease exacerbations. OMICS 2010;14:9-59.

16 Huang YJ, Erb-Downward JR, Dickson RP, et al. Understanding the role of the microbiome in chronic obstructive pulmonary disease: principles, challenges, and future directions. Trans/ Res 2017:179:71-83.

17 National Institute for Health and Clinical Excellence. Chronic obstructive pulmonary disease: management of chronic obstructive pulmonary disease in adults in primary and secondary care. London: National Clinical Guideline Centre, 2010.

18 Caporaso JG, Kuczynski J, Stombaugh J, et al. QIIME allows analysis of highthroughput community sequencing data. Nat Methods 2010;7:335-6.

19 Eriksson LJE, Kettaneh-Wold N. Multi- and megavariate data analysis, Part 2, advanced applications and method extensions: MKS Umetrics AB, 2006.

20 Flores GE, Caporaso JG, Henley JB, et al. Temporal variability is a personalized feature of the human microbiome. Genome Biol 2014;15:531.

21 R Core Team (2014) R: A language and environment for statistical computing. $R$ foundation for statistical computing. Vienna, Austria. ISBN 3-900051-07-0. http:// www.R-project.org/

22 Benjamini Y, Hochberg Y. Controlling the false discovery rate: a practical and powerful approach to multiple testing. J $R$ Statist Soc B 1995;57:289-300.

23 Hilty M, Burke C, Pedro $\mathrm{H}$, et al. Disordered microbial communities in asthmatic airways. PLoS One 2010;5:e8578

24 Erb-Downward JR, Thompson DL, Han MK, et al. Analysis of the lung microbiome in the "healthy" smoker and in COPD. PLoS One 2011:6:e16384.

25 Zakharkina T, Heinzel E, Koczulla RA, et al. Analysis of the airway microbiota of healthy individuals and patients with chronic obstructive pulmonary disease by T-RFLP and clone sequencing. PLoS One 2013;8:e68302.

26 Pragman AA, Kim HB, Reilly CS, et al. The lung microbiome in moderate and severe chronic obstructive pulmonary disease. PLoS One 2012;7:e47305.

27 Dahl M, Vestbo J, Lange $P$, et al. C-reactive protein as a predictor of prognosis in chronic obstructive pulmonary disease. Am J Respir Crit Care Med 2007;175:250-5.

28 Langille MG, Zaneveld J, Caporaso JG, et al. Predictive functional profiling of microbial communities using $16 \mathrm{~S}$ rRNA marker gene sequences. Nat Biotechnol 2013;31:814-21.

29 Worlitzsch D, Tarran R, Ulrich M, et al. Effects of reduced mucus oxygen concentration in airway Pseudomonas infections of cystic fibrosis patients. J Clin Invest 2002:109:317-25.

30 Schmidt A, Belaaouaj A, Bissinger R, et al. Neutrophil elastase-mediated increase in airway temperature during inflammation. J Cyst Fibros 2014;13:623-31.

31 Marsland BJ, Gollwitzer ES. Host-microorganism interactions in lung diseases. Nat Rev Immunol 2014;14:827-35.

32 Lopez-Campos JL, Agustí A. Heterogeneity of chronic obstructive pulmonary disease exacerbations: a two-axes classification proposal. Lancet Respir Med 2015:3:729-34

33 Wedzicha JA, Brill SE, Allinson JP, et al. Mechanisms and impact of the frequent exacerbator phenotype in chronic obstructive pulmonary disease. BMC Med 2013;11:181.

34 Hurst JR, Vestbo J, Anzueto A, et al. Susceptibility to exacerbation in chronic obstructive pulmonary disease. N Engl J Med 2010;363:1128-38. 